\title{
Use of Simulated Patients as an Educational Resource for Bioethics Education
}

\author{
Derek D'Souza
}

Director National Bioethics Curriculum Implementation Centre, MIMER Medical College, Talegaon Dabhade, Pune.

Corresponding Author: Derek D'Souza

E-mail: dsjdsouza@gmail.com

\begin{abstract}
The use of actual patients as a teaching resource in medical education has been the practice since time immemorial. Both doctors and patients have also accepted this as both necessary and irreplaceable. However, in recent times with a renewed understanding of bioethical principles relating to patients' rights and human rights there has been a paradigm shift in this equation. Simulated patients are one well accepted alternative that have certain distinct advantages as an alternative to use of real patients. Many studies have shown that real patients and SPs can make highly valued and indispensable contributions to undergraduate medical education. SPs have considerable advantages compared with real patients used as an educational resource, including their availability and flexibility. Also, SPs can be trained to provide students with feedback, which is valued by the students. Simulated patients, can provide a safe, low anxiety learning environment where students can learn from immediate feedback and their own mistakes and build their competence and confidence. This is of immense value in Bioethics education where the affective domain plays a major role in both teaching and assessment.
\end{abstract}

Key words: simulated patients, bioethics, education

\section{INTRODUCTION}

Medical education today is faced with myriad challenges and concerns for the future healthcare professionals. The knowledge base has expanded tremendously and the students are expected to absorb, assimilate and demonstrate a range of knowledge, skill and above all empathy by the time they pass out from the institution. With added emphasis on bioethics and communication training modules, the curriculum has become quite complex and challenging for the faculty who have to adapt and get the students ready for the 'real- world' of medicine. This is possible only with new approaches in the training of medical students and residents to make them ready for the future ethical and professional challenges they will encounter.

Even in India, the statutory bodies that control medical education have introduced a new mandate that required all educational programmes to include measures of competence in all domains, including ethics and professionalism.

\section{Training methodology for medical undergraduates}

For a long time, bulk of the training focus had been on demonstrable domains of professional competence, i.e., measurable knowledge and observable skills that are requisite to achieve an optimal standard of care. On the other hand, most of the ethical issues fall into the affective domain 
and this entails newer methodologies and strategies for teaching. Only if the training is comprehensive enough to include both these domains will it be truly complete. This will ensure that the highest ideals and practices of the healthcare profession are upheld [1].

There are many studies as evidence that medical students and residents are faced with ethical dilemmas that are related to their stage of training. Thus, there is a need for clinically oriented integrated bioethics education to prepare them for the day-to-day ethical problems that they are likely to encounter in daily duties [2-3].

It has to be mentioned that there are also many studies suggesting that the present form of training leads to 'traumatic de-idealisation', cynicism psychological, and moral exhaustion, which influences the ethical decision making by student doctors during their formative years [4-8].

Students enter the institution with dreams and ideals to serve humanity and save lives and be wonderful doctors. Unfortunately, as they pass through many years of medical school they witness first-hand the reality of medical practice - what actually happens in wards and inside the OT. At first, they are shocked and appalled, as time goes by, they meekly follow what they are told to do despite their own feelings and emotions. What is unacceptable is when this goes full cycle and the students now start to emulate all the wrong-doings that they had hated in the initial stages of training as they feel there is no alternative.

\section{Real Patients or Simulated Patients}

Patients are a valuable educational resource and are required for the students to understand and observe the signs and symptoms of disease. Hence, they have been traditionally used in many different educational settings, such as bedside teaching, watching surgeries in the operation theatre etc. They are an effective means to demonstrate teaching skills, such as physical examination, history taking, communication, and procedural skills [9-11]. Students have the opportunity to observe skill demonstration by senior clinicians and having the patient at the centre of teaching is the best method that could be used [11].

One of the limitations of utilising patients as an educational resource is concern among students and faculty about utilising the patients as 'guinea pigs' and also the issue of their comfort. There is a worry that bedside teaching and discussion of the treatment options would be stressful to patients [12].

One of the main criticisms of bedside teaching is the innate variability because of inherent differences between patients. This make it difficult to standardise or demonstrate the same level of signs symptoms for two batches of students over a period of time. There is also the difficulty of arranging the desired type of patients especially at the time of examination or assessment [13-17]. Another complication with the practice of using real patients is that at times they condition may deteriorate or their condition be too complicated to participate in bedside teaching. Cases of rare diseases too raise ethical issues of whether they can be made a 'public spectacle' in the name of sharing medical knowledge. In addition, actual clinical teaching is disturbed by lesser number of in-patient beds, shorter hospital stays, and reductions in the number of faculty [17-18]. There is a rising demand of OPD and indoor patients for increased numbers of fresh intake of medical, nursing students, as well as postgraduate residents [15]. Waterbury [17] has suggested that use of SPs can be an alternative to compensate for the limitations of bedside teaching.

\section{Use of Simulated Patients as an Educational Resource}

SPs are generally healthy individuals who are meticulously trained to perform the role of a patient realistically and consistently. SPs were introduced by Barrows in 1964 and they have been extensively used in medical education ever since [19-21]. There have been many studies which are evidence that SPs may be a valuable alternative to real patients $[14,16,22]$. Other advantages of SPs over real patients, as described by Barrows, are that they can be made available as per requirement after the initial training period is over, they can be safely employed, they can be taught to adapt to students' learning needs and most importantly they reduce the variation otherwise seen in normal patients [21].

The learning interaction with SPs can be organised at any time and in any setting, unlike interactions with real patients whose need to be admitted in the hospital or report to the OPD. 
Interaction with the SPs can be less embarrassing for students during their initial training when their interviewing and physical examination skills are not yet perfected. The students may be permitted to make errors especially in challenging and sensitive situations, such as the pelvic examination of female patients or breaking bad news. SP training and implementation can be adapted for specific educational purposes. For example, an interaction with a SP can be interrupted as required to clarify details regarding the case or give tips to the student. There is also the advantage that the SPs can be examined repeatedly till the students' can perfect their examination techniques. With a properly trained SP it is also possible to change the difficulty level of the patient encounter based on the competence of a particular student. In this way variability in learning can be compensated for by allowing each student to question and examine an SP who is simulating the same medical problem in the same way. SPs can simulate a wide range of physical findings, for example wheezing, abdominal tenderness, muscle weakness, and tremor [21]. Furthermore, SPs are easy to train and can contribute to training a variety of skills [16, 20-21].

People of various age groups may be trained to perform as SPs. For example, adolescent girls have been reported to highly value their performance in a patient role [23]. SPs have also been reported as being motivated by interaction with the students and cherish their role in the making of the future health professionals [24]. In turn, students too have reported to enjoy their workshops with SPs dealing with important soft skills, such as breaking bad news [22].

The students rated the workshops as effective with an almost realistic learning as well as immediate feedback from the SP. They also felt that they did not have concerns about harming the patients that they would normally have. Another significant benefit when SPs are used is the fact that they can be used to assess the students' performance as well. This is more relevant in terms of bioethics training where much of the assessment is based on soft skills appreciation and behavioural or attitudinal change. In contrast one study has reported that medical undergraduates preferred real patients to SPs because of their authenticity [25]. This might be a limitation of SPs.

The significant features of educational experiences gleaned from interaction with real patients is the presentation of actual abnormal physical findings. For example, there can be no comparison to listening to an actual cardiac murmur or palpation of an enlarged spleen or liver in an actual patient. Real patients have limitations on their utility. The reality is that there are fewer suitable patients for the teaching of medical students. This is due to the numerous changes in the practice and administration of health care. In addition, there are always the concerns related to patients' comfort and confidentiality. Being allowed to examine and interact with real patients is vital for comprehensive medical education. This is despite the recent development of many sophisticated and realistic simulation techniques. Hence it is important to understand that there is a place for both real patients and the simulated patients will always be an add-on or a value add. The aim should be to have a realistic balance between simulated patient and real patients as well as use of simulators. Both the real patients and the SPs should not feel that they are over-burdened in their designated roles.

\section{Training of SPs for Bioethics education}

Training of SPs is very important as it is not just knowledge and clinical skills that have to be evaluated but also the affective domain as regards bioethical skills development.

1. The patient first has to be made familiar with the clinical settings and the role that the simulated patient is expected to play. This can be exhibited by the help of multimedia movies demonstrations etc.

2. The SPs are initially included as subjects in class demonstrations of anatomy, clinical examination techniques, etc.

3. Observation, by SPs, of real patients being interviewed and examined, especially patients who have the clinical signs that have to be mimicked later on.

4. Interacting with the SPs to simulate a real patient's clinical complaints and perfect their actions. Their understanding and duplication of the patient findings can be refined.

5. An experienced SP who may also be a professional actress can be used at the final phase to demonstrate to the other trainee SPs to perform during the clinical examination and bedside interview. 
6. Finally, there is need for an independent evaluation and assessment of the training output of the SPs and their actual performance by an independent member of the teaching faculty who has had no contact with the training program.

7. A suitably trained simulated patient can effectively enact the symptoms of coma, seizures, paralysis, sensory losses, reflex changes, blindness, etc, as required. Signs that cannot be simulated such as papilledema, atrophy, fasciculation, etc can be described in the history and the examining medical student is expected to assume their presence. Additionally, the actual test results such as ECG's, EEG's, radiographs, EMG's and laboratory test results may be given to the students. Using this patent protocol detailed clinical instruction can be offered to medical students, using just one simulated patient and one instructor. This has immense value in areas where patients of a particular disease are not likely to be found.

The present system of assessment of a real patient examination does not permit evaluation of the student's technique or comparison between students, since they all cannot examine the same patient. This means that there may be wide variation in terms of the responses of a particular patient at any given time. In contrast, a simulated patient can present a repeatable history, in the manner of a real patient, and repeatable findings based on those of an actual patient. In addition, this simulated patient, when specially trained, can subsequently report on the details of the student's examination technique and bedside manner.

\section{Advantages of use of SPs in Bioethics education}

1. A student can rehearse the examination technique on SPs without facing any embarrassment or hesitation that a new doctor would otherwise feel in front of ill patients and without concern for tiring them, hurting them, or aggravating their disease condition.

2. The simulated patient can be examined multiple times until the student's technique is up to the expected standards without fear of harming the patient.

3. All necessary aspects of disease complications and prognosis can be openly discussed in the presence of the patients without concern for their reaction to such information. This is important in the initial stages till the students perfect their clinical and bed-side manner.

4. Actual patients who are normally utilised as subjects for clinical teaching are relieved of this responsibility and can be cared for as per their requirement.

5. A clinical case can be presented to a student in a manner so that all the relevant findings can be predetermined, thereby allowing for uniform evaluation of the student's clinical ability.

6. The same clinical problem can be presented again and again to different students, allowing for standardization of inter-student comparison and for assessment of the efficacy of the teaching program.

7. A properly trained simulated patient can also act as an assessor. They can report objectively on the student's bedside skills, physician-patient rapport, manner, approach, etc.

Some educators have objections to the use of SPs by claiming that - A simulated patient is no substitute for a real patient and that all clinical teaching should be carried out using real patients. Continued use of real patients has major drawback when considering ethical rights, human dignity and patient rights. It also fails to recognize that the role played by a simulated patient occupies an intermediary position between books, didactic teaching, multimedia tools and specimens and actual real-life patients. Now there is an alternative where the students have actual reacting humans on whom the students hone their clinical skills and at the same time be evaluated for their clinical performance.

\section{CONCLUSION}

In summary, it may be said that in addition to using actual patients in medical education, SPs may be added as valuable educational adjuncts. Most SPs have been seen to enjoy their roles as actual patients. The advantages of SPs are that they are well-disciplined and adaptable. They are readily available when required, adjustable to the requirements of the students' needs, offer uniformity of 
educational experiences across students, and enable repeated practice of skills. They also ideal in the teaching and assessment of bioethics education where much of the training is related to the affective domain.

\section{REFERENCES}

1. Wartman SA, Brock DW. The development of a medical ethics curriculum in a general internal medicine residency program. Acad Med 1989;64:751-4.

2. Mitchell JJ. Residents' desire for more clinical ethics education. Acad Med 1993:68.

3. Roberts L, Teresita M, Lyketsos C, Hardee JT, Jacobson J, Walker R. What and how psychiatry residents at ten training programs wish to learn ethics. Acad Psychiatry 1996; 20:131-43.

4. Spencer J, Blackmore D, Heard S, et al. Patient-oriented learning: a review of the role of the patients in the education of medical students. Med Educ 2000;34:851-7.

5. Self DJ, Schrader DE, Baldwin DC Jr, Wolinsky FD. The moral development of medical students: a pilot study of the possible influence of medical education. Med Educ 1993;27:26-34.

6. Self DJ, Baldwin DC Jr, Wolinsky FD. Further exploration of the relationship between medical education and moral development. Camb Q Health Ethics 1996;5:444-9.

7. Patenaude J, Niyonsenga T, Fafard D. Changes in the components of moral reasoning during students' medical education: a pilot study. Med Educ 2003;37:822-9.

8. Hayes RP, Stoudemire AS, Kinlaw K, Dell ML, Loomis A. Changing attitudes about end-of-life decision making of medical students during third-year clinical clerkships. Psychosomatics 1999; 40:205-11.

9. Janicik RW, Fletcher KE. Teaching at bedside: a new model. Med Teacher 2003;25:127-30.

10. Kroenke K, Omori DM, Landry FM, Lucey CR. Bedside teaching. S Med J 1997;90:1069-74.

11. Fitzgerald FT. Bedside teaching. West J Med 1993;158:418-20.

12. Hodgson $\mathrm{H}$. Teaching and the patient. Br Med J 1975; 1:628.

13. Nair BR, Coughlan JL, Hensley MJ. Student and patient perspectives on bedside teaching. Med Educ 1997;31:341-6.

14. McGraw RC, O'Conner HM. Standardized patients in the early acquisition of clinical skills. Med Educ 1999; 33:572-8.

15. Anderson KK, Meyer TC. The use of instructor-patients to teach physical examination techniques. J Med Educ 1978;53:831-6.

16. Stillman PL, Swanson DB. Ensuring the clinical competence of medical school graduates through standardized patients. Arch Intern Med 1987;147:1049-52.

17. Waterbury JT. Refuting patients' obligations to clinical training: a critical analysis of the arguments for an obligation of patients to participate in the clinical education of medical students. Med Educ 2001;35:286-94.

18. Farquhar D. Reducing reliance in hospitalized patients for undergraduate clinical skills teaching in internal medicine. Acad Med 2000;75:537.

19. Barrows HS, Abrahamson S. The programmed patients: a technique for appraising student performance in clinical neurology. J Med Educ 1964;39:802-5.

20. Stillman PL, Regan MD, Philbin M, Haley HL. Results of a survey on the use of standardized patients to teach and evaluate clinical skills. Acad Med 1990;65:288-92.

21. Barrows HS. An overview of the uses of standardized patients for teaching and evaluating clinical skills. Acad Med 1993;68:443-51.

22. Fortin AH, Haeseler FD, Angoff N. Teaching pre-clinical medical students an integrated approach to medical interviewing: half-day workshops using actors. J Gen Intern Med 2002;17:704 -8.

23. Blake K, Gusella J, Greaven S, Wakefield S. The risks and benefits of being a young female adolescent standardised patient. Med Educ 2006;40:26-35.

24. Davies M. The way ahead: teaching with simulated patients. Med Teacher 1989;11:315-20.

25. Simek-Downing L, Quirk ME, Letendre AJ. Simulated versus actual patients in teaching medical interviewing. Fam Med 1986;18:358-60.

$* * * * * * * * * * * * * * * * * * *$

Acknowledgements - Nil; Funding - Nil; Conflict of Interest - Nil. 